\title{
Protée
}

\section{Cindy Dumais : NOIRS \& PEAU / première ph(r)ase}

\section{Jean-François Caron}

Volume 37, numéro 2, automne 2009

Avec le génocide, l’indicible

URI : https://id.erudit.org/iderudit/038455ar

DOI : https://doi.org/10.7202/038455ar

Aller au sommaire du numéro

Éditeur(s)

Département des arts et lettres - Université du Québec à Chicoutimi

ISSN

0300-3523 (imprimé)

1708-2307 (numérique)

Découvrir la revue

Citer cet article

Caron, J.-F. (2009). Cindy Dumais : NOIRS \& PEAU / première ph(r)ase. Protée, 37(2), 57-66. https://doi.org/10.7202/038455ar d'utilisation que vous pouvez consulter en ligne.

https://apropos.erudit.org/fr/usagers/politique-dutilisation/ 


\section{CINDY DUMAIS}

NOIRS \& PEAU / première ph(r)ase

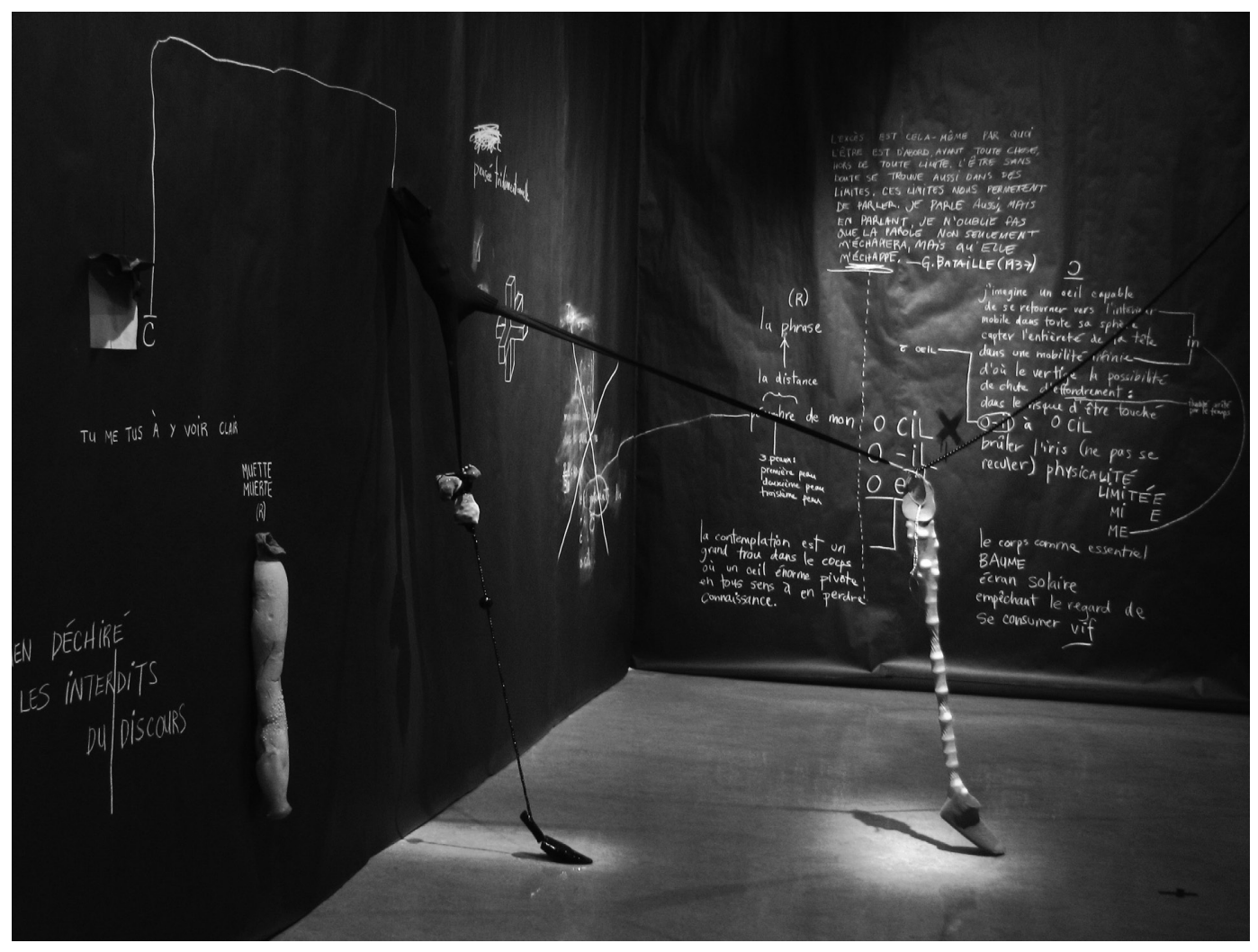




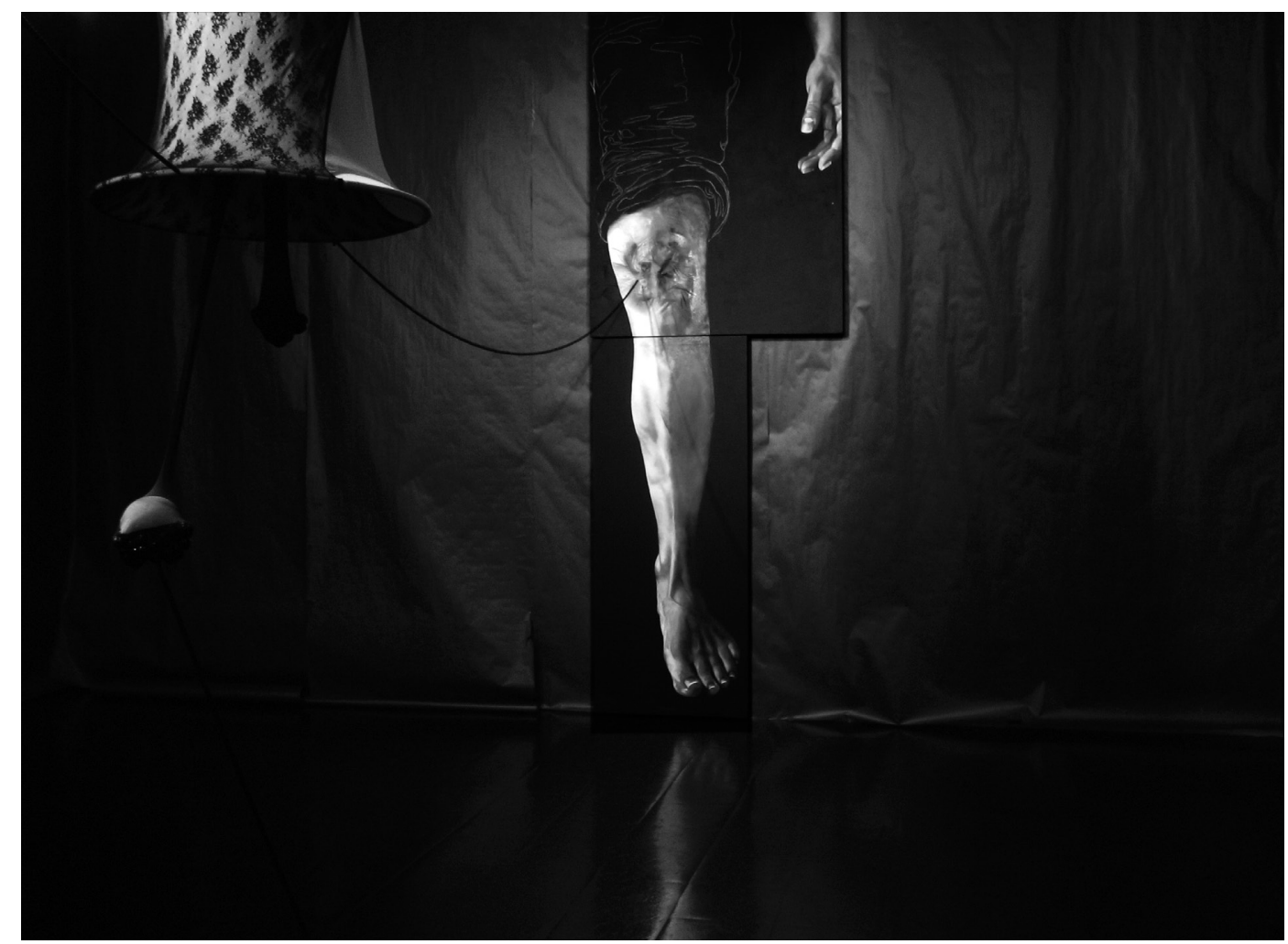

Première phase d'un large projet faisant appel à plusieurs médiums, tels que la peinture, l'écriture, la vidéo et la sculpture. NOIRS \& PEAU étudie le déboîtement, en faisant se disloquer différentes parties du corps dans la représentation. Ce déboîtement est non seulement physique, mais aussi psychique, car il était une façon de rendre manifeste cette sensation qu'est la pensée. La salle, complètement noire, dont les murs tapissés de papier portaient des inscriptions à la craie, était remplie de plusieurs objets fabriqués, prenant l'apparence de bijoux lustrés et brillants. D'une esthétique baroque, aux limites de l'ornemental, ces objets-bijoux prenaient place dans l'espace, créant ainsi une atmosphère feutrée, intrigante et enveloppante. NOIRS \& PEAU a été présenté à la galerie L'CEuvre de l'Autre du 14 janvier au 11 février 2009. 


\section{UNE OEUVRE ANTIPERSONNELLE}

C 'EST le propre du génocide que de sombrer dans l'indicible tant la souffrance est grande et le motif incompréhensible. On a sans doute tout dit de la Shoah, sans pourtant jamais I'approcher. Or, si les mots manquent invariablement leur cible, le travail de Cindy Dumais, par son ouverture à la fois sur le sens et l'insensé, transgresse les règles strictes du langage pour s'avancer sur les territoires les plus sombres de I'humanité, fauchant les jungles du senti. Le projet NOIRS \& PEAU / première ph( $r$ )ase est la première étape d'un projet monumental, mais aussi l'introduction d'un texte qui ne cesse de se réécrire.

Le caractère de ce qui est inhumain correspond non pas à ce qui est étranger à l'homme, mais plutôt à une part de lui-même - il n'y a que ce qui est en lui qui peut être in-humain. C'est donc de l'intérieur que Dumais aborde les pires souffrances (subies ou infligées), comme si elle retournait l'œil pinéal de Bataille pour trouver un soleil qui serait inhérent à la chair, grandiose et brûlant. Malgré I'aspect monumental de l'œuvre projetée par l'artiste, c'est donc un rapport très intimiste à l'Histoire qu'on y décèle. Le corps devient le transit meurtri de l'humanité et de son Histoire, alors que les voies de la souffrance se font toutes pénétrables.

Au cœur du travail de Cindy Dumais se trouve l'idée de la marque - une évidence apportée entre autres par les procédés de thermoformage et la présentation de membres amputés ou difformes. Pour l'artiste, la confrontation du corps avec le réel, la maladie ou la ligne du temps laisse des traces, qu'elles soient cicatrices ou souvenirs assassins. Par exemple, jamais la craie ne s'efface véritablement. Elle laisse des poussières de paroles avortées parmi les mots qui trouvent leur place sur les murs de l'espace d'exposition ${ }^{1}$. Même le masque de laque noire appliqué sur certains mots est révélateur ; on ne saurait plus lire le texte original, mais on a une certitude : on a voulu dissimuler. Cette stratégie n'est pas sans évoquer l'indélébilité de tous les « effacés » des registres de I'Histoire, en Allemagne, en Russie, en Bosnie ou au Rwanda. La trame historique s'accorde aux cris de ceux dont on refuse la disparition.

Dans le discours de Dumais, le sujet est en perpétuelle correspondance avec l'autre, se brûle aux mêmes bûchers, pourrit dans les mêmes charniers. Il se disloque pour laisser surgir un autre corps à travers lui-même, dans une étreinte surhumaine. Ce relais de I'humain à l'humanité se déploie comme par contamination, laissant se répandre une sombre mais précieuse humeur. Il est un rang de perles ou un bijou organique impensable, mais aussi une éclaboussure de tous les noirs, de toutes les noirceurs qui viennent de l'intérieur, une tache «trou noir» qui à la fois marque et absorbe.

Dans ce rapprochement entre le sujet et l'universel, même interdire devient un dire ensemble. Ce rassemblement humain dans un corps éclaté détonne comme un cri, comme une œuvre antipersonnelle dans une hurlante proximité. L'Histoire est comprise dans et par le corps, dont la mémoire inaltérable (mais sensible) est marquée par les souffrances qui la ponctuent. C'est pourquoi le travail de Dumais excède les possibilités du dire. Le langage de la douleur, s'il est source d'incompréhension, n'en demeure pas moins précieux : il permet d'envisager l'infigurable.

Jean-François Caron

1. L'artiste cite entre autres Georges Bataille, dont le discours sur l'excès est une influence importante. 


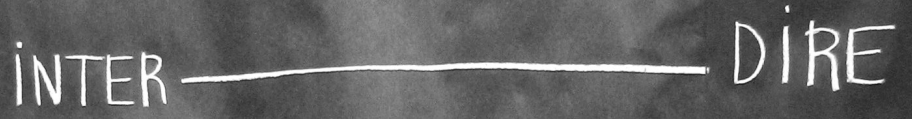

p propre
b bouche

sale

bouche

e

gants

blanc

mains

rouge

$\therefore$

$\because \therefore$

$\therefore$ 


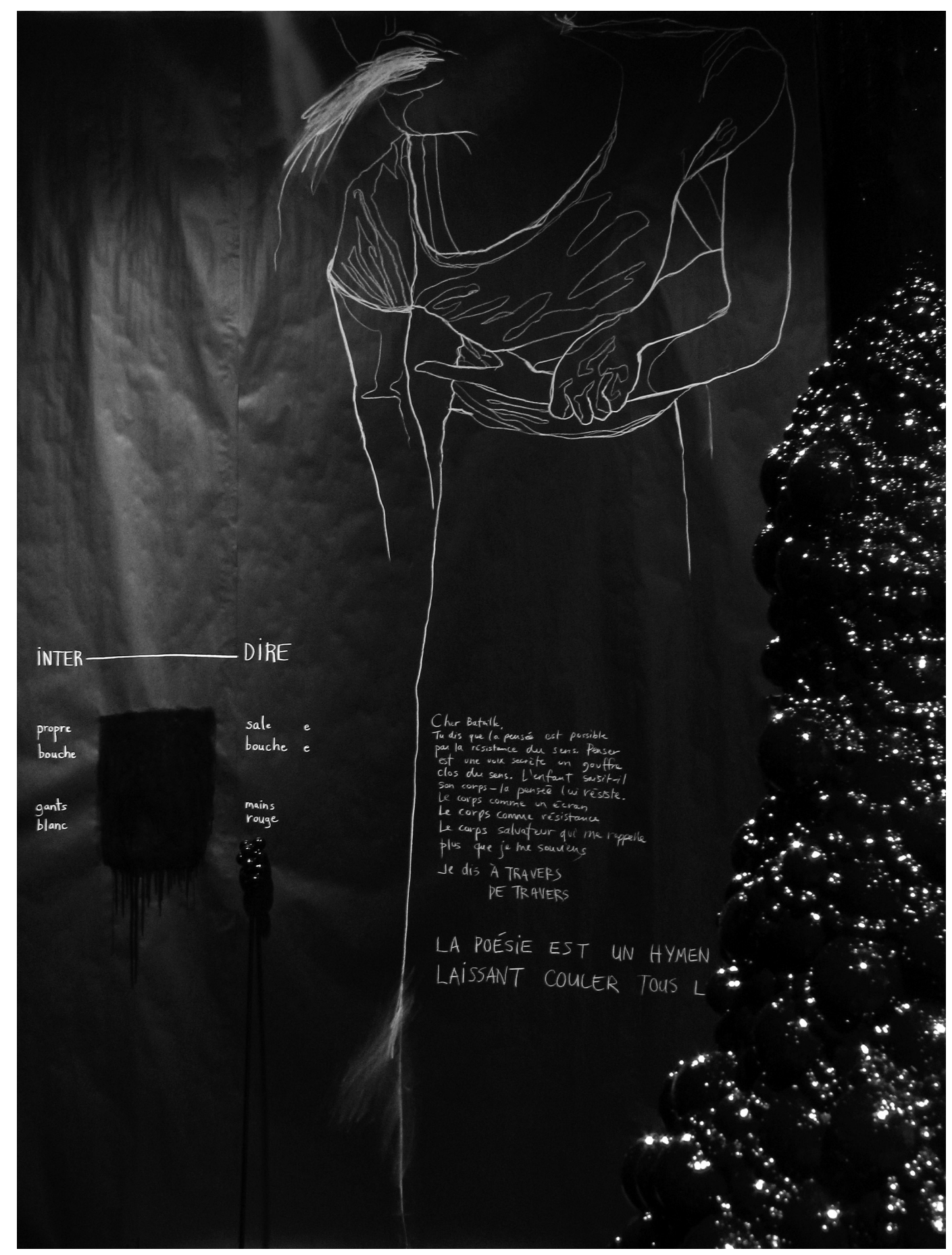




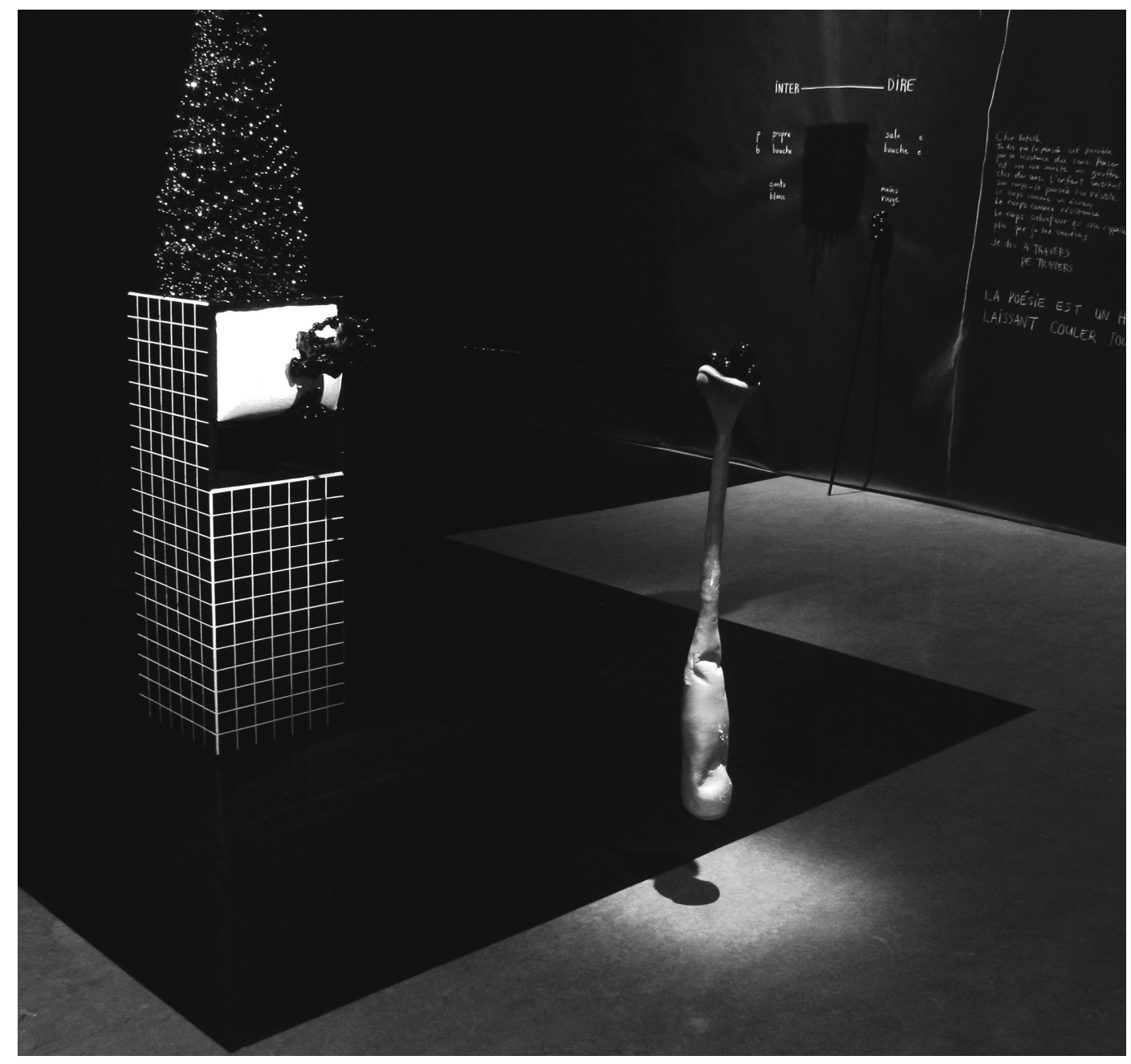




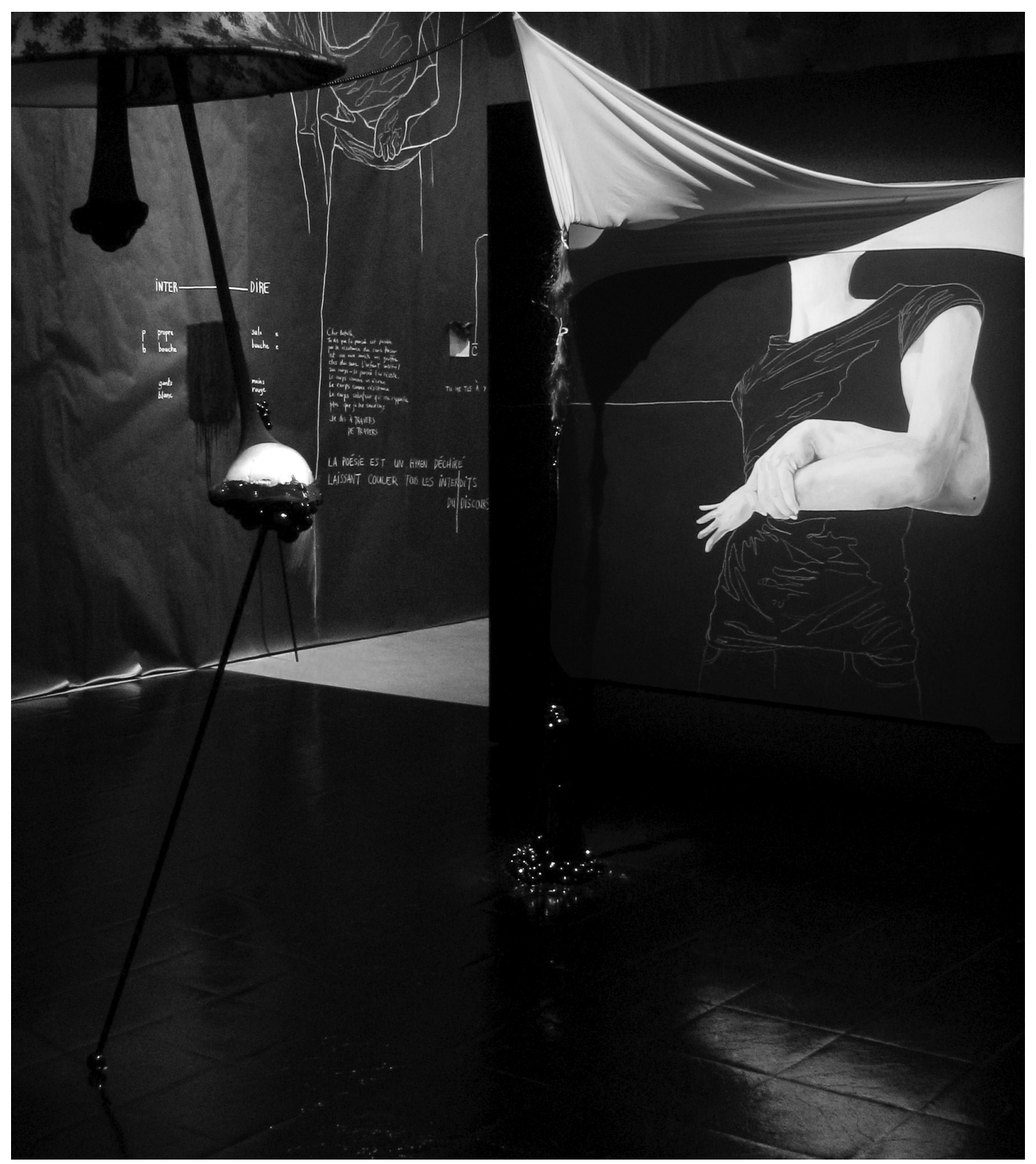




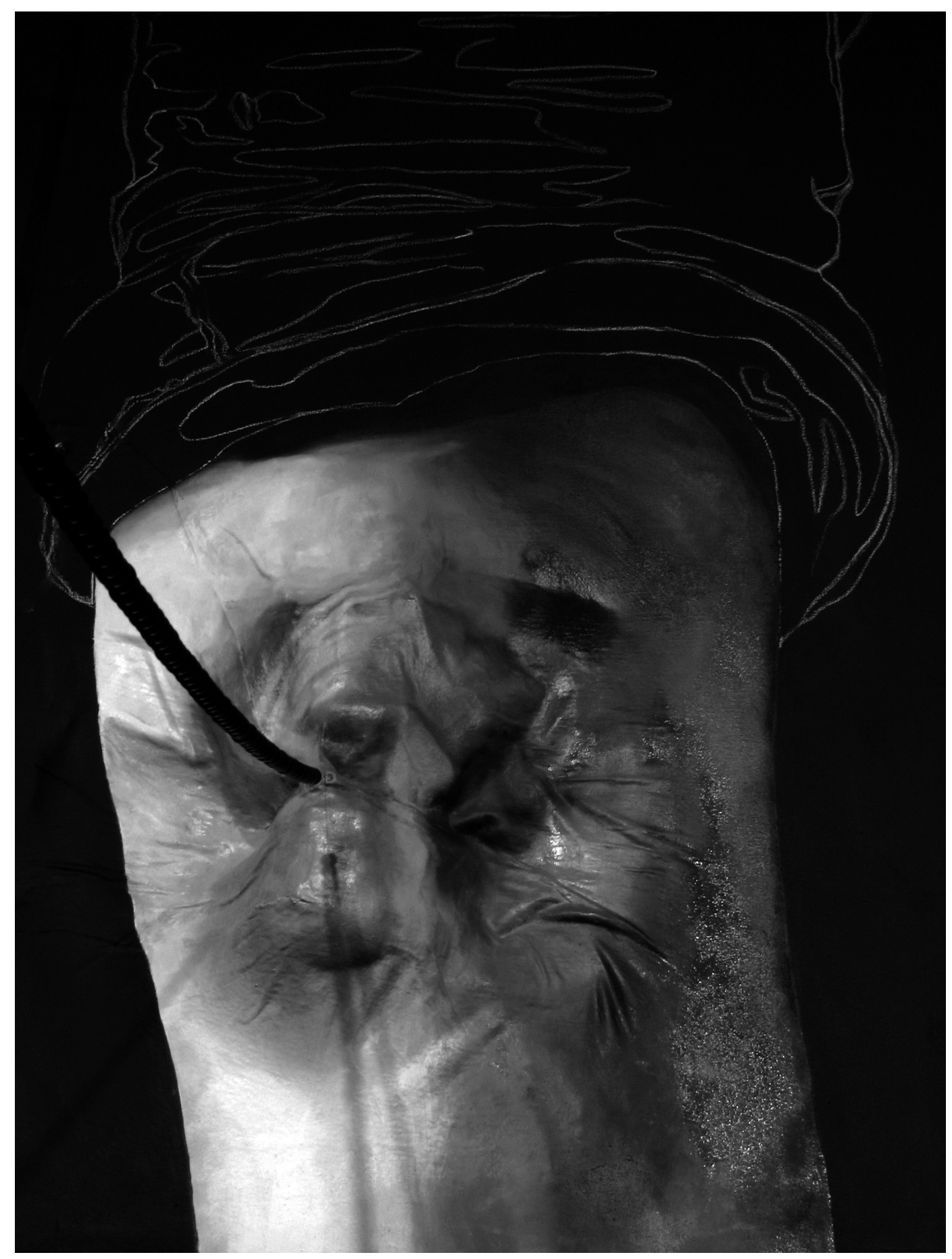




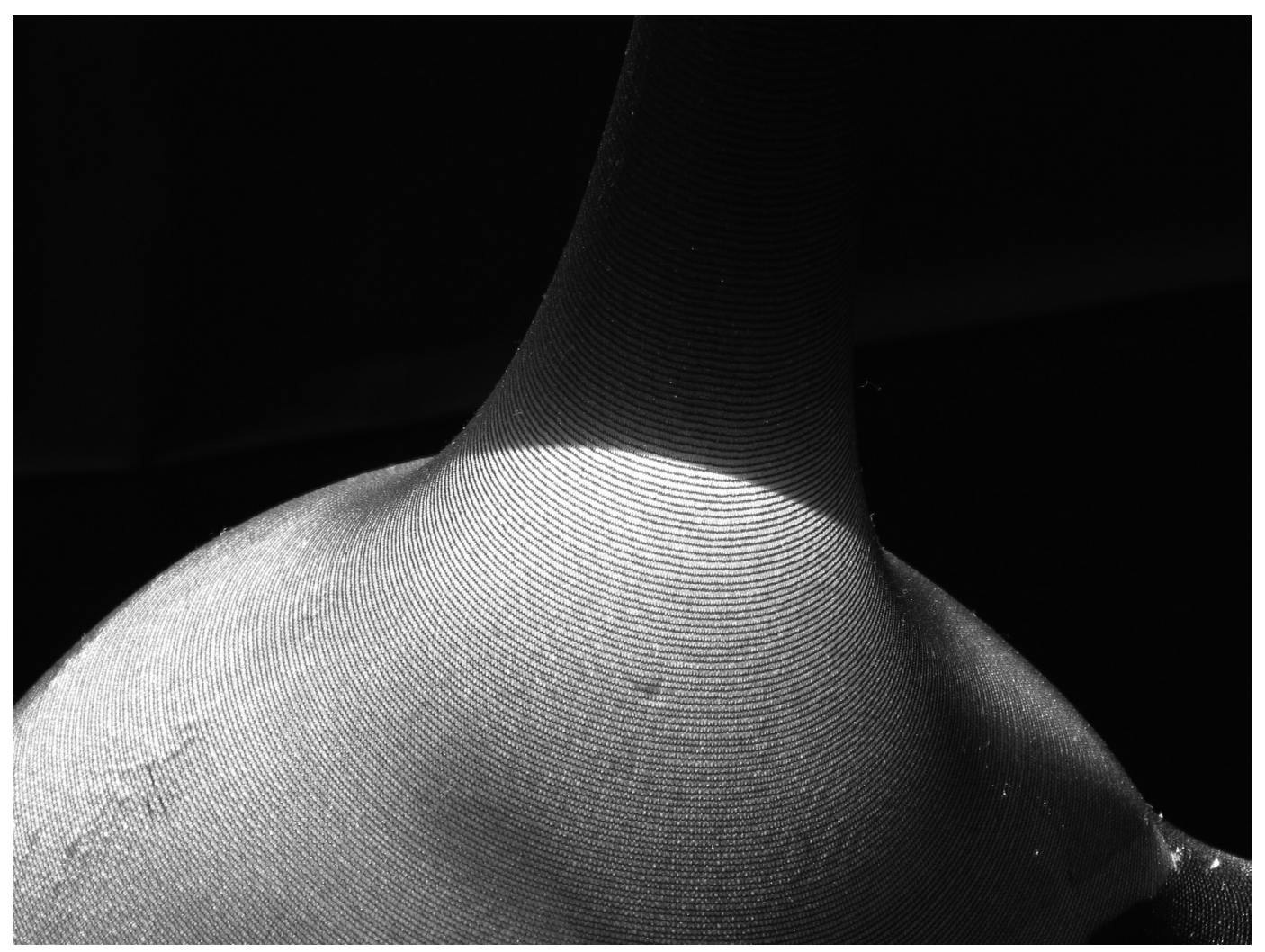




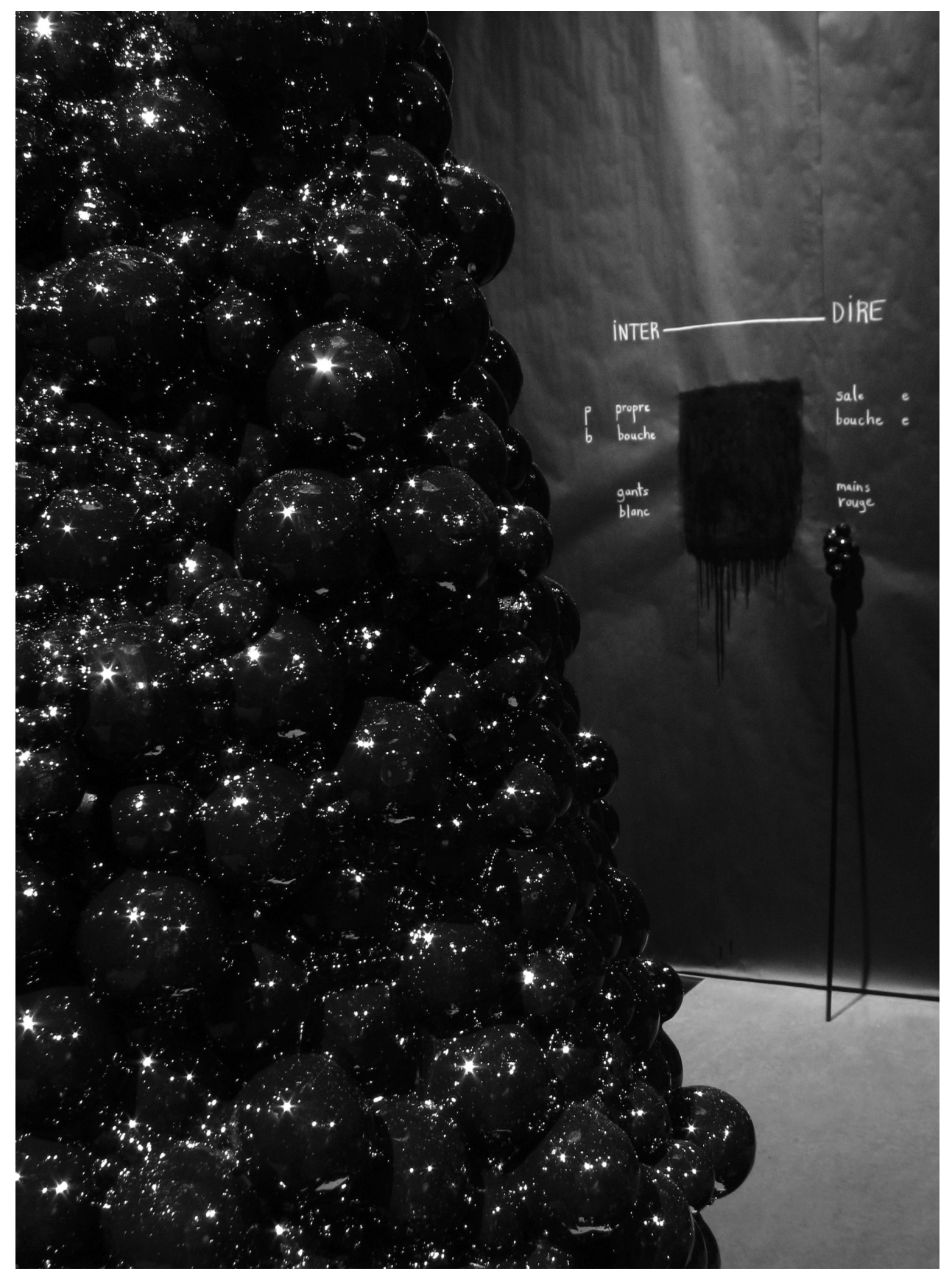

